\section{A importância dos ácidos graxos poliinsaturados de cadeia longa na gestação e lactação}

\author{
The significance of long chain \\ polyunsaturated fatty acids in pregnancy \\ and lactation
}

Deila Regina Bentes da Silva 1 Paulo Fernando Miranda Júnior 2 Eliane de Abreu Soares 3 Janeiro. Rua São Francisco Xavier, 524. $12^{\circ}$ andar, Bloco D. Rio de Janeiro, RJ, Brasil. CEP: 20.550-900.

E-mail: deilabentes@yahoo.com.br

\begin{abstract}
Long chain polyunsaturated fatty acids ( $L C$ PUFAS), docosahexaenoic acid (DHA) and arachinodonic acid (AA) are essential components for both neurological development and visual function of the child. This review focused on recent studies concerning the value of these nutrients during gestational and neonatal periods. The need for ( $L C$ PUFAS) is enhanced during intra-uterine and first months of life. The mother is the principal source of fatty acids to the child. Maternal milk contains all required nutrients for infant's growth and development. Babies do not have the ability to elong and desaturate fatty acids therefore; feeding formulas should contain preformed LC-PUFAS.
\end{abstract}

Key words Pregnancy, Lactation, Lactating, Essential fatty acids, Linoleic Acid, Linolenic acid, Docosahexaenoic acid, Arachinodonic acid

\section{Resumo}

Os ácidos graxos poliinsaturados de cadeia longa (AGPICL), ácido docosahexaenóico (DHA) e ácido araquidônico (AA) são componentes essenciais não só para o desenvolvimento neurológico quanto para a função visual da criança. $O$ objetivo desta revisão foi buscar estudos recentes sobre a importância desses nutrientes no período gestacional e neonatal. A maior necessidade dos AGPICL ocorre durante a vida intrauterina e nos primeiros meses de vida. A mãe é um fator determinante na oferta desses ácidos graxos para a criança. O leite materno contém todos os nutrientes necessários para o crescimento e desenvolvimento da criança. $O$ bebê não tem capacidade de elongação $e$ dessaturação dos ácidos graxos e dessa maneira as fórmulas infantis devem conter AGPICL pré-formados.

Palavras-chave Gestação, Lactação, Lactente, Ácido graxo essencial, Ácido linoléico, Ácido linolênico, Ácido docosahexaenóico, Ácido araquidônico 


\section{Introdução}

A importância dos lipídios na nutrição e desenvolvimento humano é reconhecida há muitas décadas. Os ácidos graxos (AG) são constituintes estruturais das membranas celulares, cumprem funções energéticas e de reservas metabólicas, além de formarem hormônios e sais biliares. ${ }^{1}$ Dentro da diversidade dos AG, existem aqueles que o organismo tem capacidade de síntese, porém outros não. Esses AG cuja biossíntese é inadequada são denominados ácidos graxos essenciais (AGE): ácido linolênico ( $\omega$-3) e ácido linoléico ( $\omega-6)$. Para suprir a demanda orgânica, os mesmos devem estar em quantidades suficientes na alimentação. Vários estudos apontam que sua utilização traz benefícios para a saúde humana, prevenindo enfermidades cardiovasculares, câncer de cólon, doenças imunológicas e favorecendo o desenvolvimento cerebral e da retina. ${ }^{1-4}$

O $\omega$-6 e o $\omega-3$ são considerados precursores dos ácidos graxos poliinsaturados de cadeia longa (AGPICL): Ácido araquidônico (AA), ácido eicosapentaenóico (EPA), e ácido docosahexaenóico (DHA). 3 O ácido araquidônico (série $\omega$-6) tem grande importância nos primeiros meses de vida, sendo constituinte de estruturas celulares e precursores de mediadores inflamatórios. 5 O DHA (série $\omega$-3) é considerado o AGPICL mais importante no desenvolvimento neonatal e juntamente com o AA são os principais componentes dos AG cerebrais. 1,6

Nos últimos anos, diversos estudos têm investigado a importância dos AGPICL na alimentação do recém-nascido para obter o máximo potencial de desenvolvimento neurológico. Portanto, eles são considerados nutrientes fundamentais para o perfeito desenvolvimento cerebral e visual do bebê antes e após o nascimento. 5,7-10 A inquestionável notabilidade dos AGE e de seus derivados estimulou a elaboração desta revisão, cujo objetivo foi buscar na literatura as informações recentes sobre a relação daqueles nutrientes no período gestacional e neonatal. Foi realizada uma pesquisa bibliográfica, utilizando as seguintes palavras-chave: gestação, lactação, lactente, ácidos graxos essenciais, ácido graxo linoléico, ácido graxo linolênico, ácido docosahexaenóico, ácido araquidônico. Para o acesso às bases de dados foram pesquisados os Periódicos Capes, Bireme e MD Consult, no período de 1999 a 2004 tendo sido selecionadas publicações de estudos randomizados.
Importância dos AGPICL durante a gestação e

lactação

\section{Gestação}

A dieta materna, antes da concepção, é de grande importância, já que ela determina o tipo de ácido graxo que se acumulará no tecido fetal.2,5 O transporte dos AGE é realizado através da placenta e são depositados no cérebro e retina do concepto. Além disso, ocorre um acúmulo simultâneo nas glândulas mamárias durante esta fase. ${ }^{11} \mathrm{O}$ depósito de DHA na retina e no córtex cerebral ocorre principalmente no último trimestre de gestação e nos primeiros seis meses de vida extra-uterina, ${ }^{11,12}$ podendo se estender até os dois primeiros anos de vida. 1,10

O consumo de pescados e a suplementação com óleo de pescados podem reduzir a incidência de parto prematuro e melhorar o peso do bebê ao nascer. ${ }^{2}$ Além disso, o conteúdo de AGPICL no cordão umbilical se correlaciona diretamente com o consumo destes ácidos graxos pela mãe. ${ }^{7}$

$O$ feto não tem a capacidade de sintetizar AGPICL através de seus precursores $\omega-3$ e $\omega-6$, tendo a sua necessidade suprida unicamente pela placenta. Assim como o fígado fetal, esse anexo não tem atividade biossintética de elongação e dessaturação para formar AGPICL. Durante o último trimestre de gestação, a placenta estabelece preferência no transporte de DHA e AA, pelas maiores necessidades.6,13 $\mathrm{O}$ aporte dos AGPICL deve ser garantido pelas reservas tissulares da mãe, principalmente do tecido adiposo. 1,4

$\mathrm{Na}$ gestação há situações que podem alterar o aporte desses ácidos como: nutrição inadequada, consumo de gordura e óleos com alta proporção de $\omega-6$ e muito baixo aporte de $\omega$-3, o que é muito comum, gestações freqüentes e múltiplas que podem diminuir consideravelmente as reservas de AGPICL. 1

Dessa forma, se a mãe receber uma alimentação com um aporte adequado de AGPICL, poderá oferecer ao feto a quantidade necessária desses ácidos para um bom desenvolvimento do sistema nervoso e visual. Além disso, a própria gestação caracteriza-se como um período vulnerável para a deficiência desses ácidos, devendo a gestante ingerí-los em sua dieta para satisfazer não só as necessidades do concepto como também as suas.

\section{Lactação}

Após o nascimento, o lactente continua incapaz de sintetizar os AGPICL, devido à imaturidade hepática estar presente. Porém a placenta é substituída pelo leite materno como meio de oferta desses AG. 7 
Durante a lactância a mãe continua a oferecer o aporte de AGPICL. O leite materno apresenta três vezes mais AA e DHA que o leite de vaca, sendo o segundo insuficiente para atender as necessidades do lactente. Os neonatos mais vulneráveis para desenvolver deficiência de AGPICL são os recémnascidos pré-termo e os que são alimentados com fórmulas industrializadas sem a presença desses ácidos graxos. Os AGPICL são essenciais em prematuros com pouca reserva lipídica. Pela limitada reserva calórica, os mesmos deverão mobilizar parte dos ácidos graxos para atender suas necessidades quando o aporte exógeno for inadequado. Isso poderá ocasionar transtornos, como: crescimento inadequado, dermatites, aumento da susceptibilidade de infecções, entre outras. 6

Os prematuros alimentados com fórmulas enriquecidas com AGPICL apresentaram concentrações elevadas de DHA e AA nos eritrócitos 14,15 e no plasma15 quando comparados com aqueles alimentados com fórmulas convencionais (sem adição de AGPICL), mas apresentaram concentrações inferiores em relação aos prematuros alimentados com leite humano.14,15

Comparando a concentração dos AGPICL no cordão umbilical de recém-nascido pré-termo e a termo, Araya et al.16 observaram que as concentrações de AA e DHA foram significantemente menores em pré-termo $(p<0,05)$. Provavelmente o aporte nutricional com AGPICL deverá ser diferente para essas crianças.

O desempenho nos testes de desenvolvimento dos prematuros alimentados com fórmulas suplementadas é melhor do que daqueles com fórmulas sem adição. $15 \mathrm{O}$ índice de desenvolvimento psicomotor mostrou-se superior nessas crianças, porém o índice de desenvolvimento mental e acuidade visual não apresentaram o mesmo efeito. ${ }^{17}$

O enriquecimento com AGPICL favorece o ganho de peso 18,19 e de comprimento ${ }^{19}$ de crianças prematuras sem apresentar nenhum efeito adverso. ${ }^{18,19}$

Com a diminuição da idade gestacional, aumenta a imaturidade de certos tecidos, entre eles o cérebro. Dessa forma, a oferta desses AGPICL em quantidades adequadas é primordial, principalmente para os grupos mais vulneráveis em apresentar a deficiência desses ácidos graxos, sendo o leite humano, indiscutivelmente, o alimento mais indicado durante os primeiros meses de vida. ${ }^{1}$

\section{Fontes de AGPICL}

As principais fontes de DHA e EPA são os pescados, moluscos, algas e crustáceos. 1,2,4 Dessas, o pescado, principalmente o jurel (espécie de pescado encontrado no Chile), atum, anchova, sardinha e salmão, 4 fornecem uma maior quantidade desses nutrientes. Porém, a variação no conteúdo de AG $\omega$-3 dos alimentos marinhos dependerá da espécie do pescado, parte comestível, local de captura, época do ano, temperatura da água e do processo de industrialização. Essa variação pode chegar até $25 \% .^{2}$

No âmbito mundial, o consumo de pescado vem diminuindo desde os anos 50 e dessa forma o consumo de $\omega$-3 também. Isso poderia não ser importante pelo fato de que os seres humanos têm a capacidade de converter o $\omega$-3 que se encontra nos vegetais verdes e em certos óleos vegetais em EPA e DHA. Porém este processo de conversão não é muito eficiente e está sujeito a uma inibição competitiva por parte dos AG $\omega-6$. As dietas modernas proporcionam sete, oito ou 10 vezes mais $\omega-6$ que $\omega-3.2$

Outras fontes de $\omega-3$ presentes na dieta são alguns frutos oleaginosos, sementes, vegetais, gema de ovo, polvo e carnes de ruminantes. Existem muitos produtos no mercado que vêm sendo enriquecidos com AG $\omega-3$, por exemplo ovo, azeites, leite, fórmulas infantis, entre outros enriquecidos com diferentes quantidades desse ácido. Dos produtos, o ovo é o mais comumente disponível, já que a ração dos frangos é enriquecida com $\omega$-3.2 Não é demais recordar que o melhor aporte nutricional de ácidos graxos $\omega$-3 e $\omega$-6 são obtidos através de uma alimentação rica em vegetais e produtos do mar, os quais lamentavelmente cada vez consumimos menos. 4

Em estudos experimentais, Visentainer et al.20 pesquisaram a concentração de EPA e DHA em peixes marinhos da costa brasileira e demonstraram que a quantidade total de EPA e DHA foi maior para as espécies sardinha e bonito, sendo considerados boas fontes desses ácidos, especialmente a sardinha, por ser de baixo custo e ter grande disponibilidade no mercado nacional.

\section{Importância dos AGPICL para o desenvolvimento e função do sistema nervoso e visual}

O ácido docosahexaenóico (DHA) é fundamental para o desenvolvimento cerebral e visual do recémnascido, 5,10,12,13 pois o mesmo é componente estrutural dos fosfolipídios das membranas celulares, particularmente da fosfatidilcolina, fosfatidiletanolamina e fosfatidilserina. Devido ao seu alto grau de poliinsaturação, o DHA confere à membrana uma grande fluidez, sendo essa essencial para que as proteínas possam ter a mobilidade necessária para 
desempenhar suas funções na camada bilipídica. $\mathrm{Na}$ formação do tecido cerebral e visual, a fluidez das membranas é particularmente importante. ${ }^{1,4}$

\section{Desenvolvimento do sistema nervoso}

O desenvolvimento do sistema nervoso, especialmente do cérebro, ocorre durante o último trimestre de gestação. Durante esse período, a necessidade de DHA aumenta consideravelmente e a mãe exerce um papel fundamental na oferta não apenas de DHA como de AA, uma vez que uma baixa concentração deste no cérebro e na retina pode repercutir na funcionalidade dos órgãos afetados. ${ }^{1,4}$

O cérebro é um tecido principalmente lipídico, com cerca de $60 \%$ de seu peso seco, dos quais $40 \%$ são de AGPI e desses cerca de $10 \%$ são de AA e $15 \%$ de DHA. ${ }^{1}$

Os AGPI têm função primariamente estrutural. ${ }^{3}$ Este processo morfogênico tem início na crista neural, se caracterizando por sucessivas etapas de neurogênese, migração neural, apoptoses seletivas, sinaptogênese e mielinização. Essas etapas seqüenciais dão forma e funcionalidade do tecido cerebral. ${ }^{1}$ A deficiência de DHA pode alterar a composição das membranas sinápticas, afetando as funções dos receptores da membrana neuronal, canais iônicos e enzimáticos. 3

Alguns estudos comprovam que bebês alimentados exclusivamente ao seio materno apresentam maiores concentrações de DHA no tecido cerebral, quando comparados com os alimentados com fórmulas sem DHA.7,21,22 Isso pode explicar a correlação positiva desses ácidos graxos com uma maior capacidade de aprendizagem e maior poder de concentração, avaliado por testes específicos aplicados meses depois de terminada a lactância. ${ }^{1}$

\section{Desenvolvimento do sistema visual}

No sistema nervoso central, a retina apresenta concentrações elevadas de DHA, principalmente no último trimestre de gestação. Ao mesmo tempo, as concentrações de AA diminuem com o amadurecimento da retina, sobretudo de fosfatidiletanolamina. ${ }^{6}$

As altas concentrações de DHA encontram-se nas membranas dos cones e bastonetes, conferindolhes a fluidez necessária para que ocorra o processo de transdução do sinal luminoso (fotoexcitação da rodopsina) e sua conversão em sinal elétrico, que é processado posteriormente pelo cérebro. $1,4,12$

\section{Influência dos ácidos graxos trans na saúde materno-infantil}

Os ácidos graxos trans-isômeros são transferidos da mãe para o feto. Experimentos animais sugerem que a alta ingestão de ácidos graxos trans (AGT) inibe a formação dos AGPICL. A concentração de AGPICL no cordão umbilical de crianças a termo mostrou-se inversamente proporcional à concentração de AGT.23,24 Além disso foi inversamente relacionado com o peso ao nascer. ${ }^{23}$ A concentração de AGT no leite de lactantes também foi inversamente relacionado com a concentração de $\omega-6$ e $\omega-3.25$

Os ácidos graxos trans-isômeros encontram-se associados com o desenvolvimento da préeclâmpsia, contração uterina e infarto do miocárdio. Pequena quantidade de AGT está relacionada com a prematuridade. Mulheres com pré-eclâmpsia que fazem uso de dietas ricas neste tipo de ácido graxo, apresentam $30 \%$ mais AGT nas células vermelhas do sangue quando comparadas com mulheres sem préeclâmpsia. 26 Portanto parece ser necessário o controle da ingestão desse tipo de ácido graxo, principalmente durante a gestação.

\section{Oferta de AGPICL durante a gestação e lactação}

O aporte adequado de AGPICL durante o período gestacional e pós-natal influencia no desenvolvimento do sistema nervoso e visual do recémnascido,1,27,28 além de poder ter repercussão na inteligência e na intelectualidade do indivíduo na vida adulta.4,29

A maior necessidade de DHA ocorre durante a vida intra-uterina, especialmente durante o último trimestre de gestação e nos primeiros meses de vida. 1,4,10,28 Diante disso, o aumento da oferta destes nutrientes deve ser feito desde a gestação, onde a mãe oferta AGPI ao feto pelo transporte placentário até o recém-nato pela amamentação. 1,30

Através de várias pesquisas observou-se que a oferta de DHA em gestantes 31 e lactantes aumenta significativamente a concentração desse ácido graxo no leite, 12,21 no plasma 21,31 e eritrócitos 15,31 sem promover redução de $\omega-6.31$

Estudo randomizado 32 mostrou que, após duas semanas, a suplementação materna de DHA (200 $\mathrm{mg} /$ dia) durante o período de lactação foi capaz de dobrar o conteúdo do mesmo no leite em relação ao grupo placebo $(p=0,003)$. É sugerido que uma oferta de $300 \mathrm{mg} /$ dia de DHA na gestação seria adequada para atender as recomendações nesse período 33 e que uma oferta acima de $200 \mathrm{mg} / \mathrm{dia}$ de DHA na lactação seria capaz de aumentar a concen- 
tração desse ácido graxo no leite. ${ }^{7}$

A suplementação na lactação apenas com AA tende a diminuir a concentração de DHA e EPA no leite. No entanto, a suplementação com AA+DHA+EPA leva a um aumento da concentração desses AGPICL na secreção láctea. 34

Gaete et al.,12 em um estudo randomizado, com lactantes, verificaram que o aumento do consumo de alimentos ricos em DHA elevou em 46,6\% o conteúdo desse ácido graxo no leite. $\mathrm{Na}$ dieta materna utilizou-se o jurel (pescado típico do Chile) que para nós brasileiros, assemelha-se à sardinha e ao xerelete. A quantidade oferecida de pescado ficou em torno de $160 \mathrm{~g}$ duas vezes por semana durante 14 dias, sendo que $100 \mathrm{~g}$ de parte comestível do jurel fornece $621 \mathrm{mg}$ de DHA.

Foi demonstrado que crianças alimentadas ao seio apresentaram melhores coeficientes intelectual, acuidade visual e capacidade de adaptação à luz. ${ }^{2}$

A suplementação materna com DHA pode não apresentar efeito imediato no desenvolvimento do sistema visual do bebê logo após o nascimento. Porém, pode influenciar no desenvolvimento visual precoce de crianças a termo. 27 Crianças de gestantes e lactantes que foram suplementadas com DHA+EPA, a partir da $18^{\mathrm{a}}$ semana de gestação até o terceiro mês pós-parto, mostraram desenvolvimento mental tardio favorável.28

A necessidade de incrementar AGPICL na alimentação de gestantes e lactantes deve-se principalmente ao consumo inadequado de alimentos fontes desses nutrientes na dieta. Por isso, vários medicamentos a base de óleo de peixes e/ou derivados surgiram, principalmente no mercado internacional. 20 Além da estratégia medicamentosa, o enriquecimento de alimentos (carnes, aves e leite de vaca) seria uma outra alternativa para correção desse problema. ${ }^{35}$ Entretanto, a educação nutricional pode intervir de maneira decisiva no consumo de alimentos marinhos, aumentando assim a ingestão de AGE, seus derivados e de outros nutrientes. Essa prática foi adotada em um estudo realizado no Chile com lactantes, que após participarem de ações educativas, aumentaram o consumo de jurel. A conseqüência desse aumento interferiu positivamente no aumento desses ácidos graxos no leite, sem diminuir as concentrações de $\mathrm{AA}^{7}$ (Tabela 1).

\section{AGPICL no leite humano}

O leite materno é a maior fonte de energia, ácido graxo essencial e de vitaminas para o lactente.5,36 Ele contém mais de 150 diferentes ácidos, dentre os quais $\omega-6, \omega-3$, AA, DHA e vários outros da série $\omega-3$ e $\omega-6$, que correspondem 15 a $20 \%$ do total de ácidos graxos presentes. $37 \mathrm{O}$ leite humano apresenta boa biodisponibilidade de DHA e AA, conferindo um melhor aproveitamento desses ácidos aos bebês amamentados ao seio, quando comparados com aqueles alimentados com fórmulas lácteas. 22 A quantidade de DHA presente no leite materno varia entre 0,1 a $1,4 \%$ do total de ácidos graxos.7,21 Isso depende da dieta materna, sendo maior em regiões com alto consumo de pescados, como por exemplo os países do norte da Europa. 12,21

Inúmeras pesquisas publicadas nos últimos cinco anos mostraram que a composição do leite de mulheres ocidentais geralmente tem $10 \%$ - $17 \%$ de $\omega-6 ; 0,8-1,4 \%$ de $\omega-3 ; 0,3-0,7$ de AA e $0,1-0,5$ $\%$ de DHA.15,25,34,38 Estudos em outras áreas do mundo demonstram concentrações altas de DHA e AA no leite humano. No Japão as concentrações ficaram em torno de 1,1 e 1,0\% respectivamente, 39 enquanto na China 2,8 \% de DHA. 37 Esse fato pode ser explicado pela alta ingestão de peixe e frutos do mar dessas populações. $39 \mathrm{Na}$ Austrália36 e no Canadá, 37 foi observado um declínio no conteúdo de DHA no leite, provavelmente pelo baixo consumo de alimentos fontes de DHA e AA.

Schmeits et al.,5 analisando a composição do leite de mulheres do Nepal, observaram que a proporção de $\omega-6$ foi muito baixa em relação ao leite de outras mulheres do mundo $(8,2-17,2 \%)$. Porém, a proporção de AA ficou na média $(0,35 \%)$. A concentração de $\omega$-3 no leite de mulheres nepalense foi aproximadamente duas vezes maior $(1,93 \%)$ quando comparada a de outras mulheres do mundo.

Vários fatores podem afetar a composição lipídica no leite, incluindo tempo pós-parto, período do dia, dieta da mãe, paridade, situação socioeconômica e presença de desordem metabólica e infecções 5,36 (Tabela 2).

\section{Aporte de AGPICL a partir de fórmulas enriquecidas}

É sabido que o leite humano é a melhor forma de assegurar a oferta de AGPICL para o bebê, mas quando a prática da amamentação é impossibilitada, o uso de fórmulas infantis aparece como uma alternativa para a alimentação do lactente. Apesar do progresso tecnológico, o objetivo de se aproximar da composição do leite humano ainda está muito distante de ser conseguido. ${ }^{1}$

Pela limitada capacidade hepática de alongar e dessaturar os AGE, os AGPICL (AA e DHA) devem estar presentes na composição da fórmula láctea.7 Várias fontes de lipídeos podem ser utilizadas no 
Tabela 1

Estudos controlados com grávidas e lactantes utilizando suplementação de ácidos graxos poliinsaturados.

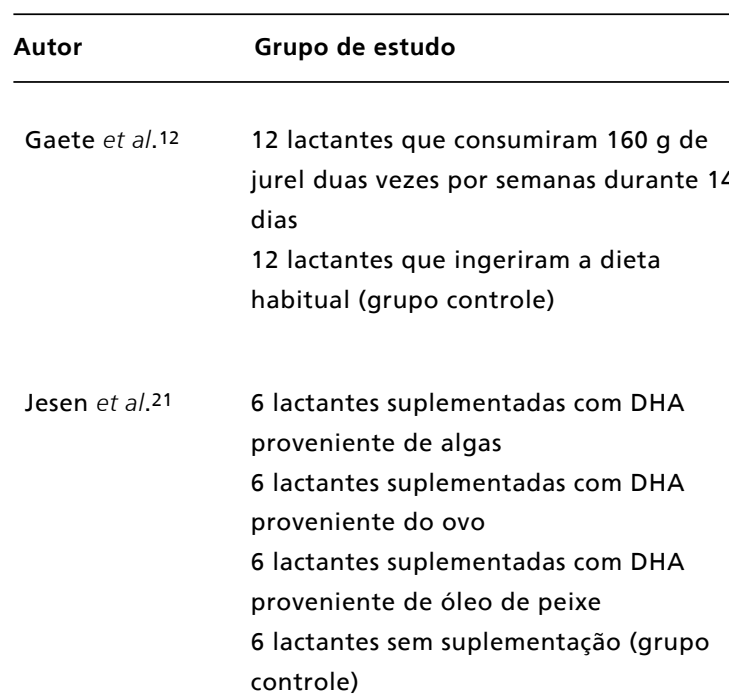

Otto et al.31

12 grávidas com suplementação de DHA e AA por quatro semanas

12 grávidas sem suplementação (grupo controle)

Filder et al.32

Gaete \&

Atalah7

Smit et al.34

10 lactantes suplementadas com $300 \mathrm{mg}$

AA durante uma semana

8 lactantes suplementadas com $300 \mathrm{mg}$

$\mathrm{AA}+110 \mathrm{mg}$ EPA e $400 \mathrm{mg}$ DHA

durante uma semana diariamente

8 lactantes sem suplementação diária

Malcolm et al.27 50 gestantes suplementadas com cápsula enriquecida em DHA desde a $15^{\mathrm{a}}$ semana de gestação até a concepção

50 gestantes sem suplementação mg de DHA/dia de quatro a seis semanas pós-parto

5 lactantes sem suplementação

24 lactantes com incentivo ao consumo
Análise da composição de DHA no plasma e no leite por cromatografia líquido-gasosa

Coleta de sangue para análise da composição de ácidos graxos dos fosfolipídios plasmáticos e dos eritrócitos

Análise da composição lipídica do leite por cromatografia líquido-gasosa

Análise da composição lipídica do leite pela transesterificação direta

Analise da composição lipídica do leite por cromatografia líquido-gasosa

Análise da composição do DHA nos eritrócitos na $15^{\mathrm{a}} / 28^{\mathrm{a}}$ e $40^{\mathrm{a}}$ semana de gestação, e no momento da concepção por cromatografia líquidogasosa, e teste de acuidade visual

\section{Principais resultados}

A suplementação materna com alimentos ricos em DHA, como o jurel, aumentou o conteúdo de DHA no leite

A suplementação materna com DHA aumentou a concentração de DHA no plasma e no leite materno do grupo que usou a suplementação com DHA

Aumento da concentração no plasma e nos eritrócitos do grupo que usou suplementação, sem redução do nível de $\omega-6$

A suplementação com DHA na dieta aumentou o seu conteúdo no leite materno

Aumento do conteúdo de DHA no leite quando a ingestão do mesmo for maior que 200 $\mathrm{mg} / \mathrm{dia}$.

A suplementação com AA diminui a concentração de DHA e EPA no leite e a combinação de AA + EPA e DHA aumentaram a concentração desses na secreção láctea

A suplementação materna com DHA não aumentou a concentração do mesmo nos eritrócitos, porém pode influenciar no desenvolvimento visual precoce de crianças a termo

DHA = ácido docosahexaenóico; $\mathrm{AA}$ = ácido araquidônico; EPA = ácido eicosapentaenóico. 
Estudos controlados avaliando a composição dos ácidos graxos poliinsaturados no leite materno.

\begin{tabular}{llll}
\hline Autor & Grupo de estudo & Metodologia & Principais resultados \\
\hline Schmeits et al.5 & $\begin{array}{l}48 \text { lactantes da zona } \\
\text { rural do Nepal }\end{array}$ & $\begin{array}{l}\text { Análise da composição lipídica do } \\
\text { leite por cromatografia gasosa }\end{array}$ & $\begin{array}{l}\text { Baixa concentração de } \omega \text { - } 6 \text { e alta concentração } \\
\text { mulho leite quando comparadas com }\end{array}$ \\
& & & mutras nacionalidades
\end{tabular}

Innis e Elias 37

55 mulheres

canadenses no período

de gestação e lactação

Questionário de freqüência alimentar

na $28^{\mathrm{a}}$ e $35^{\mathrm{a}}$ semana de gestação.

Análise plasmática de $\omega-3$ e $\omega-6$ na $35^{\text {a }}$

semana gestacional.

Análise da composição de DHA no

leite

Wang et al.39 20 nutrizes japonesas

Análise da composição lipídica do leite por cromatografia líquido-gasosa
A concentração de DHA plasmático correlacionou-se positivamente com a ingestão de alimentos fontes.

Baixo nível de DHA no leite de mulheres canadenses por provável baixo consumo de alimentos fontes em DHA

Alta concentração de DHA e AA no leite pelo alto consumo de alimentos fontes

DHA = ácido docosahexaenóico; $\mathrm{AA}$ = ácido araquidônico

enriquecimento, como exemplo: óleos de peixe, algas, fungos, ovos e triglicerídeos sintetizados.

Sala-Vila et al.,22 em estudo randomizado, observaram que independente da fonte utilizada no enriquecimento de fórmulas infantis, seja na forma de triglicerídeos sintetizados a partir de células isoladas de algas e fungos ou na forma de fosfolipídio do ovo, baixas concentrações de EPA e DHA foram encontradas no plasma de crianças alimentadas com essas, mostrando que a biodisponibilidade entre as fontes utilizadas no enriquecimento foi semelhante.

Alguns estudos que avaliaram o índice de desenvolvimento infantil têm demonstrado vantagens na utilização de fórmulas fortificadas com AGPICL em relação às fórmulas padrão. $9,13,40,41$ Outros, porém, não observaram diferenças no desenvolvimento infantil utilizando fórmulas com ou sem AGPICL. 38,42,43

A fortificação de fórmulas infantis com DHA + AA ( $0,36 \%$ e $0,72 \%$ respectivamente) foi associada com um aumento de sete pontos na média de índice de desenvolvimento mental $(105,6 \pm 11,8$ versus $98,3 \pm 8,2)(p<0,05)$ em relação ao grupo que recebeu a fórmula não fortificada. 9

Com o objetivo de avaliar os efeitos da ingestão de AGPICL no crescimento infantil, acuidade visual, desenvolvimento psicológico, mental e cognitivo,
Austead et al.38 acompanharam bebês a termo alimentados com fórmulas infantis, que continham ou não AA+DHA durante um ano. As fontes utilizadas na fortificação das fórmulas foram: ovo, óleo de peixe e fungos, com quantidades similares às encontradas no leite humano. Foi observado que o desenvolvimento infantil mostrou-se semelhante entre os grupos que receberam ou não as fórmulas fortificadas.

Lucas et al. 43 compararam um grupo de crianças que receberam fórmulas enriquecidas com DHA e AA $(0,32 \%$ e $0,30 \%$ do total de ácidos graxos respectivamente) com outro grupo que não recebeu a fórmula enriquecida. Nenhum efeito benéfico foi encontrado no desenvolvimento cognitivo e motor dessas crianças. Em um outro estudo randomizado, não se observou diferença significativa na acuidade visual, mental, cognitiva e motora durante o primeiro ano de vida de crianças que receberam fórmulas só com DHA $(0,35 \%)$ ou AA+DHA (ambos fornecendo $0,34 \%$ do total de ácidos graxos) e o grupo placebo. Entretanto, aos dois anos de idade, aquelas amamentadas ao seio (grupo de referência) apresentaram melhor desenvolvimento mental em relação aos outros dois grupos que receberam as fórmulas enriquecidas. 42

Têm-se sugerido que essas diferenças nos resultados obtidos podem ser explicadas por diferentes 
Tabela 3

Estudos controlados com grávidas e lactantes utilizando suplementação de ácidos graxos poliinsaturados.

\begin{tabular}{|c|c|}
\hline Autor & Grupo de estudo \\
\hline Sala-Vila et al.22 & $\begin{array}{l}11 \text { lactentes alimentados com leite } \\
\text { humano contendo } 0,4 \text { e } 0,3 \mathrm{~g} \text { de AA e } \\
\text { DHA respectivamente } \\
12 \text { lactentes suplementados com } \\
\text { fórmula enriquecida com DHA e AA } \\
\text { proveniente dos fosfolipídios contidos } \\
\text { no ovo } \\
12 \text { lactentes suplementados com } \\
\text { fórmula enriquecida com DHA e AA } \\
\text { proveniente dos triglicerídeos } \\
\text { sintetizados de células de algas e fungos } \\
\text { (Ambas as fórmulas continham } 0,4 \text { e } 0,1 \\
\text { g/100g total de AA e DHA } \\
\text { respectivamente) }\end{array}$ \\
\hline Birch et al.9 & $\begin{array}{l}19 \text { lactentes com suplementação de DHA } \\
\text { e AA dentro dos primeiros cinco dias de } \\
\text { vida até a } 17^{a} \text { semana. } \\
17 \text { lactentes com suplementação de DHA } \\
20 \text { lactentes sem suplementação (grupo } \\
\text { controle) }\end{array}$ \\
\hline Austead et al.38 & $\begin{array}{l}80 \text { lactentes com fórmulas contendo AA } \\
\text { + DHA originadas do ovo com níveis } \\
\text { similares as encontradas no leite } \\
\text { humano durante um ano } \\
82 \text { lactentes com fórmulas contendo AA } \\
\text { + DHA originadas de óleo de peixe ou } \\
\text { fungo } \\
77 \text { lactentes com fórmula sem } \\
\text { suplementação (grupo controle) }\end{array}$ \\
\hline
\end{tabular}

Hoffman et al.13 61 lactentes que foram suplementados ou não com fórmulas contendo DHA e AA

Lucas et al. 43

Makrides42

Makrides 8 fórmulas contendo DHA AA 155 lactentes sem suplementação de referência)

23 lactentes suplementadas com fórmulas contendo DHA 24 lactentes suplementadas com fórmulas contendo DHA e AA 21 lactentes sem suplementação

36 lactentes alimentados com fórmulas

Metodologia
138 bebês amamentados ao seio (grupo 46 crianças amamentadas ao seio (grupo de referência) contendo $\omega 6 / \omega 3$ na proporção de 10:1 37 lactentes alimentados com fórmulas contendo $\omega 6 / \omega 3$ na proporção de 5:1
A análise da fração lípidica no plasma por cromatografia gasosa

Teste de desenvolvimento mental infantil segundo escala de Bayley (BSID-II)

Teste de desenvolvimento mental e psicomotor infantil segundo escala de Bayley e teste de acuidade visual.

Análise dos ácidos graxos nos eritrócitos por cromatografia e teste de acuidade visual

Teste de desenvolvimento psicomotor infantil segundo escala de Bayley

Principais resultados

Independente da fonte utilizada no enriquecimento de fórmulas infantis, baixas concentrações de EPA e DHA foram encontradas no plasma de crianças alimentadas com essas fórmulas enriquecidas quando comparadas com as amamentadas ao seio.

Melhor índice de desenvolvimento mental de lactentes que foram suplementados em relação aos que não receberam suplementação

Não foram observadas diferenças no desenvolvimento infantil utilizando fórmulas com ou sem suplementação

Observou-se melhor desenvolvimento infantil quando os lactentes utilizaram fórmulas fortificadas com DHA E AA.

Não observaram diferenças no desenvolvimento cognitivo e motor dos lactentes

Teste de desenvolvimento mental e psicomotor infantil segundo escala de Bayley e teste de acuidade visual

Análise da concentração dos AG no plasma e eritrócitos por cromatografia gasosa
Não observaram diferenças no desenvolvimento mental, crianças utilizando as fórmulas com ou sem suplementação cognitivo, motor e visual das
Alta concentração de DHA no plasma e eritrócito dos lactentes que foram alimentados com fórmula na razão de 5:1 quando comparados com os alimentados na razão de 10:1

$\mathrm{DHA}=$ ácido docosahexaenóico; $\mathrm{AA}=$ ácido araquidônico; $\mathrm{AG}=$ ácidos graxos 
fontes de AGPICL utilizadas, diferentes quantidades de AA, diferentes razões de AA: DHA, conteúdo de DHA no leite, método usado na avaliação, ou do teor de $\omega-3$ na fórmula controle. 10,40

Crianças alimentadas com fórmulas na proporção de $\omega$-6: $\omega$-3 de 5:1 tiveram grandes concentrações de DHA no plasma e nos eritrócitos em relação às alimentadas com fórmulas que continham proporção de 10:1. Porém, quando comparadas com as que eram alimentadas ao seio as concentrações de DHA plasmática e eritrocítica eram inferiores. ${ }^{8}$ Fórmulas para crianças a termo deveriam conter no mínimo 0,20\% de DHA e 0,35\% de AA do total de ácidos graxos presentes. Para pré-termo, esses valores ficariam em torno de $0,35 \%$ de DHA e $0,40 \%$ de AA. 44

Dependendo das quantidades e proporções entre os nutrientes utilizados no enriquecimento, os resultados no desenvolvimento infantil podem se aproximar daqueles obtidos com aleitamento materno exclusivo. 45

A suplementação deve ser vista com cautela, pois dependendo das quantidades e tipos de AGPICL, pode-se inibir a conversão de $\omega$-6 em ácido araquidônico ou o alto teor de EPA pode competir com o AA pela incorporação de fosfolipídios nos tecidos.5,15,31 Portanto, as fórmulas devem conter, preferencialmente, AA e DHA, sendo que as quantidades devem ser próximas daquelas encontradas no leite materno1,21 (Tabela 3).

\section{Referências}

1. Valenzuela AB, Nieto SK. Ácidos grasos omega-6 y omega3 en la nutrición perinatal: su importância em el desarrolo del sistema nervioso y visual. Rev Chil Pediatr. 2003; 74: 149-57.

2. Gonzáles MI. Ácidos grasos omega 3: benefícios y fuentes. Interciencia. 2002; 27: 128-36.

3. Hornstra G. Omega-3 long-chain polyunsaturated fatty acids and health benefits. Neuilly-sur-seine: Nutriscience Roche Vitamins; 2002.

4. Valenzuela AB, Nieto MS. Acido docosahexaenoico (DHA) en el desarrolo fetal y en la nutrición materno-infantil. Rev Med Chile. 2001; 129: 1203-11

5. Schmeits BL, Cook JA, Vanderjagt DJ, Magnussen MA, Bhatt SK, Bobik EG, Huang YS, Glew RH. Fatty acid composition of the milk lipids of women in Nepal. Nutr Res. 1999; 19: 1339-48.

6. Corria V. Deficiência de ácidos grasos esenciales en el feto y en el recién nacido pretérmino. Rev Cuba Pediatr. 2001; 73: 43-50.

7. Gaete MG, Atalah ES. Niveles de LC-PUFA n-3 en la leche materna después de incentivar el consumo de alimentos marinos. Rev Chil Pediatr. 2003; 74: 158-65.

\section{Conclusões}

Os efeitos benéficos dos AGE, particularmente dos AGPICL, para a saúde humana são evidentes. Durante a gestação, período neonatal e toda a etapa de crescimento do bebê, a oferta desses ácidos graxos em quantidades adequadas é fundamental para o bom desenvolvimento e funcionamento do cérebro e da retina. Por essa razão, a mãe desempenha um papel primordial na oferta desses ácidos graxos, devendo consumir uma dieta rica em alimentos fontes, para atender não só as necessidades da criança em cada uma dessas etapas como também as suas. A ausência de lactância materna pode produzir carência desses nutrientes por parte do recém-nato como também transtornos no seu desenvolvimento. Além disso, os AGE desempenham uma função muito importante na prevenção e tratamento de diversas enfermidades, como doenças cardiovasculares, câncer de cólon, doenças imunológicas, entre outras.

Alguns estudos observaram vantagens no desenvolvimento infantil com a utilização de fórmulas fortificadas com AGPICL. Porém, não resta dúvida que o leite materno é a melhor e mais adequada forma de ofertar esses ácidos graxos para o bebê. Além disso, mais investigações são necessárias para averiguar com maior precisão os efeitos da utilização de fórmulas fortificadas no desenvolvimento das crianças.

8. Makrides M, Neumann MA, Jeffrey B, Lien EL,Gibson RA. A randomized trial of different ratios of linoleic to alpha-linolenic acid in the diet of term infants: effects on visual function and growth. Am J Clin Nutr. 2000; 71: 1209

9. Birch EE, Garfield S, Hoffman DR, Uauy R, Birch DG Randomized controlled trial of early dietary supply of longchain polyunsaturated fatty acids and mental development in term infants. Dev Med Child Neurol. 2000; 42: 174-81.

10. Innis SM, Gilley J, Werker J. Are human milk long-chain polyunsaturated fatty acids related to visual and neural development in breast-fed term infants? J Pediatr. 2001; 139: $532-7$

11. Hayat L, Al-Sughayer M, Afzal M. A comparative study of fatty acids in human breast milk and breast milk substitutes in Kuwait. Nutr Res. 1999; 19: 827-41.

12. Gaete MG, Atalah ES, Araya JA. Efecto de la suplementación de la dieta de la madre durante la lactancia con ácidos grasos omega 3 en la composición de los lípidos de la leche. Rev Chil Pediatr. 2002; 73: 239-47. 
13. Hoffman DR, Birch EE, Castañeda YS, Fawcett SL, Wheaton DH, Birch DG, Uauy R. Visual function in breastfed term infants weaned to formula with or without longchain polyunsaturates at 4 to 6 months: a randomized clinical trial. J Pediatr. 2003;142: 669-77

14. Araya AJ, Fernández MP, Rojas GM, Pacheco AM, RoblesVV. Ácidos grasos poliinsaturados de cadena larga en eritrocitos de prematuros alimentados por vía enteral con leche humana de pretérmino o con una fórmula convencional. Rev Chil Pediatr. 1999; 70: 100-6.

15. Lima LM, Thomas AP. Lipídios em terapia nutricional pediátrica. Rev Bras Nutr Clin. 2001;16: 135-8.

16. Araya AJ, Fernández MP, Rojas GM, Mateluna AA. Ácidos grasos essenciales en eritrocitos de sangre umbilical de recién nacidos prematuros y de término, pequeños o adecuados a la edad gestacional. J Pediatr (Rio J). 2000; 76: 45-50.

17. O'Connor DL, Hall R, Adamkin D, Auestad N, Castillo M, Connor WE, Connor SL, Fitzgerald K, Groh-Wargos S, Hartmann EE, Jacobs J, Jandwsky J, Lucas A, Margeson D, Mena P, Neuringer M, Nesin M, Singer L, Stephenson T, Szabo J, Zemon, V. Growth and development in preterm infants fed long-chain polyunsaturated fatty acids: a prospective, randomized controlled trial. Pediatrics. 2001;108: 359-71.

18. Innis SM, Adamkin DH, Hall RT, Kalhan SC, Lair C, Lim M, Stevens DC, Twist PF, Diersen DA, Harris CL, Merkel KL, Hansen JW. Docosahexaenoic acid and arachidonic acid enhance growth with no adverse effects in preterm infants fed formula. J Pediatr. 2002; 140: 547-4.

19. Fewtrell MS, Abbott RA, Kennedy K, Singhal A, Morley R, Caine E, Jamieson C, Cockburn F, Lucas A. Randomized, double-blind trial of long-chain polyunsaturated fatty acid supplementation with fish oil and borage oil in preterm infants. J Pediatr. 2004;144: 471-9.

20. Visentainer JV, Carvalho PO, Ikegaki M, Park YK. Concentração de ácido eicosapentaenóico (EPA) e ácido docosahexaenóico (DHA) em peixes marinhos da costa brasileira. Cien Tecnol Aliment. 2000; 20: 90-3.

21. Jensen CL, Maude M, Anderson RE, Heird WC. Effect of docosahexaenoic acid supplementation of lactating women on the fatty acid composition of breast milk and maternal and infant plasma phospholipids. Am J Clin Nutr. 2000; 71 (Suppl): 292S-9S.

22. Sala-Vila A, Castellote AI, Campoy C, Rivero M, Rodriguez-Palmero M, López-Sabater MC. The source of long-chain PUFA in formula supplements does not affect the fatty acid composition of plasma lipids in full-term infants. J Nutr. 2004; 134: 868-73.

23. Elias SL, Innis SM. Infant plasma trans, $n-6$, and n-3 fatty acids and conjugated linoleic acids are related to maternal plasma fatty acids, length of gestation, and birth weight and length. Am J Clin Nutr. 2001; 73: 807-14.

24. Decsi T, Burus I, Molnár S, Minda H, Veitl V. Inverse association between trans isomeric and long-chain polyunsaturated fatty acids in cord blood lipids of full-term infants. Am J Clin Nutr. 2001; 74: 364-8.
25. Innis SM, King DJ. Trans fatty acids in human milk are inversely associated with concentrations of essential all-cis $\mathrm{n}-6$ and $\mathrm{n}-3$ fatty acids and determine trans, but not $\mathrm{n}-6$ and $\mathrm{n}-3$, fatty acids in plasma lipids of breast-fed infants. Am J Clin Nutr. 1999; 70: 383-90.

26. Stender S, Dyerberg J. Influence of trans fatty acids on health. Ann Nutr Metab. 2004; 48: 61-8.

27. Malcolm CA, McCulloch Dl, Montgomery C, Shepherd A, Weaver LT. Maternal docosahexaenoic acid supplementation during pregnancy and visual evoked potential development in term infants: a double blind, prospective, randomised trial. Arch Dis Child Fetal Neonatal Ed. 2003; 88: 383-90.

28. Helland IB, Smith L, Saarem K, Saugstad OD, Drevon CA. Maternal supplementation with very-long-chain $n-3$ fatty acids during pregnancy and lactation augments children`s IQ at 4 years of age. Pediatrics. 2003; 111: 39-44.

29. Crawford M. Placental delivery of arachidonic and docosahexaenoic acids: implications for the lipid nutrition of preterm infants. Am J Clin Nutr. 2000; 71: 275-84.

30. Montogomery C, Speake BK, Cameron A, Sattar N, Weaver LT. Maternal docosahexaenoic acid supplementation and fetal accretion. Br J Nutr. 2003; 90: 135-45.

31. Otto SJ, Van Houwelingen AC, Hornstra G. The effect of supplementation with docosahexaenoic and arachidonic acid derived from single cell oils on plasma and erythrocyte fatty acids of pregnant women in the second trimester. Prostaglandins Leukot Essent Fatty Acids. 2000; 63: 323-8.

32. Filder N, Sauerwald T, Pohl A, Demmelmair H, Koletzko B. Docosahexaenoic acid transfer into human milk after dietary supplementation: a randomized clinical trial. J Lipid Res. 2000; 41: 1376-83.

33. Simopoulos A, Leaf A, Salem N. Essentiality and recommended dietary intakes of omega- 6 and omega- 3 fatty acids. Ann Nutr Metab. 1999; 43: 127-30.

34. Smit EN, Koopmann M, Boersma ER, Muskiet FA. Effect of supplementation of arachidonic acid (AA) or a combination of AA plus docosahexaenoic acid on breastmilk fatty acid composition. Prostaglandins Leukot Essent Fatty Acids. 2000; 62: 335-40.

35. Valenzuela AB, Sanhueza JC, Nieto SK. Acidos grasos omega-3 de cadena larga em la nutrición humana y animal: un modelo de alimentos funcionales. Rev Chil Nutr. 2000; 27: 345-51.

36. Mena PN. Suplementación nutricional en lactancia materna. Rev Chil Pediatr. 2002; 73: 225-8.

37. Innis SM, Elias S1. Intakes of essential n-6 and n-3 polyunsaturated fatty acids among pregnant Canadian women. Am J Clin Nutr. 2003; 77: 473-8.

38. Austead N, Halter R, Hall RT, Blatter M, Bogle ML, Burks W, Erickson JR, Fitzgerald KM, Dobson V, Innis SM, Singer LT, Montalto MB, Jacobs JR, Qiu W, Bornstein MH. Growth and development in terms infants fed long-chain polyunsatured fatty acids: a double-masked, randomized, parallel, prospective, multivariate study. Pediatrics. 2001; 108: 372-81. 
39. Wang L, Shimizu Y, Kanero S, Hanaka S, Abe T, Shimasaki $\mathrm{H}$, Hisak H, Nakajima H. Comparison of the fatty acid composition of total lipids and phospholipids in breast milk from Japanese women. Pediatr Int. 2000; 42: 14-20.

40. Heird WC. The role of polyunsaturated fatty acids in term and preterm infants and breastfeeding mothers. Pediatr Clin North Am. 2001; 48: 173-88.

41. Uauy R, Hoffman DR, Mena P, Lianos A, Birch EE. Term infants studies of DHA and AA supplementation on neurodevelopment: results of randomized controlled trials. J Pediatr. 2003; 143: 17-25

42. Makrides M, Neumann MA, Simmer K, Gibson RA. A critical appraisal of the role of dietary long-chain polyunsaturated fatty acids on neural indices of term infants: a randomized, controlled trial. Pediatrics. 2000; 105: 32-8.
43. Lucas A, Stafford M, Morley R, Abbott R, Stephenson T, MacFadynen U, Elias-Jones A, Clements H. Efficacy and safety of long-chain polyunsaturated fatty acid supplementation of infant-fornula milk: a randomized trial. Lancet. 1999; 354: 1948-54.

44. Koletzko B, Agostoni C, Carlson SE, Clandinin T, Hornstra G, Neuringer M, Uauy R, Yamashiro Y, Willatts P. Long chain polyunsaturated fatty acids (LCPUFA) and perinatal development. Acta Paediatr. 2001; 90: 460-4.

45. Rodriguez A, Raederstorff D, Sarda P, LauretC, Mendy F, Descomps B. Preterm infant formula supplementation with alpha linolenic acis and docosahexaenoic acid. Eur J Clin Nutr. 2003; 57: 727-34.

Recebido em 15 de agosto de 2006

Versão final apresentada em 24 de abril de 2007

Aprovado em 30 de abril de 2007. 